\title{
ANÁLISIS BIBLIOMÉTRICO SOBRE TURISMO DE GOLF Y SOSTENIBILIDAD
}

\author{
Jesús Manuel López-Bonilla \\ Doctor \\ Profesor de la Universidad de Sevilla, España \\ lopezbon@us.es \\ Luis Miguel López-Bonilla \\ Doctor \\ Profesor de la Universidad de Sevilla, España \\ luismi@us.es \\ María del Carmen Reyes-Rodríguez
Doctora \\ Profesora de la Universidad de Sevilla, España \\ mcreyes@us.es
}

Resumen

Objetivo: El objetivo de este trabajo fue analizar los estudios realizados sobre turismo de golf desde una perspectiva de la sostenibilidad.

Método: Se ha realizado un análisis bibliométrico consultando las bases de datos Scopus y Web of Science. Se han seleccionado 46 artículos que han sido publicados en revistas científicas durante los últimos 22 años.

Originalidad / Relevancia: El turismo de golf forma parte del turismo deportivo y representa un gran auge en todo el mundo en las últimas décadas. Sin embargo, la investigación académica sobre turismo de golf ha mostrado un interés creciente hace relativamente poco tiempo.

Resultados principales: los hallazgos del análisis indicaron una alta concentración de estudios e investigadores de países mediterráneos y, concretamente, situados en el sur de la Península Ibérica, destacando las regiones del Algarve (Portugal) y especialmente Andalucía (España).

Contribuciones teóricas / metodológicas: Es el primer estudio bibliométrico sobre la temática. El turismo de golf tiene una gran importancia económica en la industria turística. Sin embargo, es necesario considerar también la influencia de la planificación y el desarrollo territorial, tanto a nivel medioambiental como social.

Palabras clave: Bibliometría. Turismo de golf. Sostenibilidad. Scopus. Web of Science.

\section{$\underline{\text { Cite como }}$}

American Psychological Association (APA)

López-Bonilla, J.M., López-Bonilla, L.M., \& Reyes-Rodríguez, M.C. (2021, jan./abr.). Análisis bibliométrico sobre turismo de golf y sostenibilidad. PODIUM Sport, Leisure and Tourism Review, São Paulo, 10(1), 56-79.

https://doi.org/10.5585/podium.v10i1.16738. 


\section{BIBLIOMETRIC ANALYSIS ON GOLF TOURISM AND SUSTAINABILITY}

\section{Abstract}

Objective: The objective of this work was to analyse the studies on golf tourism from a sustainability perspective.

Method: A bibliometric analysis has been carried out. The Scopus and Web of Science databases have been consulted. Forty-six articles have been selected which have been published in scientific journals during the last 22 years.

Originality / Relevance: Golf tourism is part of the sports tourism and represents an enormous boom around the world in recent decades. However, academic research on golf tourism has shown increasing interest in a relatively short time.

Main results: The analysis findings indicated a high concentration of studies and researchers from Mediterranean countries and, specifically, located in the south of the Iberian Peninsula, highlighting the regions the Algarve (Portugal) and especially Andalusia (Spain).

Theoretical / methodological contributions: Golf tourism is of great importance for the tourism industry. However, it is also necessary to considerer the influence of territorial planning and development, both environmentally and socially.

Keywords: Bibliometrics. Golf tourism. Sostenibility. Scopus. Web of Science.

\section{ANÁLISE BIBLIOMÉTRICA SOBRE TURISMO DE GOLFE E SUSTENTABILIDADE}

\section{Resumo}

Objetivo do estudo: O objetivo desta pesquisa foi analisar estudos sobre turismo de golfe a partir de uma perspectiva da sustentabilidade.

Metodologia/ abordagem: Uma análise bibliométrica foi realizada na base de dados Scopus e Web of Science. Foram selecionados 46 artigos que foram publicados em revistas científicas nos últimos 22 anos.

Originalidade / Relevância: $\mathrm{O}$ turismo de golfe faz parte do turismo esportivo e representa um grande boom mundial nas últimas décadas. No entanto, a pesquisa acadêmica sobre o turismo de golfe mostrou um interesse crescente em um tempo relativamente curto.

Principais resultados: Os resultados da análise indicaram uma alta concentração de estudos e pesquisadores de países mediterrâneos e, especificamente, localizados no sul da Península Ibérica, destacando as regiões do Algarve (Portugal) e, especialmente, da Andaluzia (Espanha).

Contribuições teóricas / metodológicas: O trabalho é o primeiro estudo bibliométrico sobre esse assunto. O turismo de golfe tem grande importância econômica para a indústria do turismo. 
No entanto, também é necessário considerar a influência do planejamento e desenvolvimento territorial, tanto ambiental quanto socialmente.

Palavras-chave: Bibliometria. Turismo de golfe. Sustentabilidade. Scopus. Web of Science.

\section{Introducción}

El Turismo de Golf forma parte del turismo deportivo, que es uno de los sectores más importantes desde hace ya algunas décadas (Ramírez-Hurtado \& Berbel-Pineda, 2015; Del Campo et al., 2018; Song, Chen \& Chen, 2020) y de más rápido crecimiento en la industria turística (Mason \& Moretti, 2015). El Comité Olímpico Internacional (COI) y la Organización Mundial de Turismo (OMT) suscribieron un acuerdo de cooperación en 1999, en el que ambas instituciones declararon de manera explícita la importancia que supone la relación entre el turismo y el deporte (Latiesa y Paniza, 2006). La Organización Mundial del Turismo (2019: 55) define el turismo deportivo como "un tipo de actividad turística que se refiere a la experiencia viajera del turista que o bien observa como espectador, o bien participa activamente, en un evento deportivo que implica por lo general actividades comerciales y no comerciales de naturaleza competitiva".

El golf ha experimentado un crecimiento considerable en el sector turístico europeo en los últimos años (Pastor et al., 2015). En concreto, en algunas regiones del Sur de Europa se ha adoptado el turismo de golf como un importante foco de atracción de la demanda turística. Este crecimiento se ha dado también en todo el mundo (Correia, Barros \& Silvestre, 2007) y se espera que siga en aumento gracias al impulso de los Juegos Olímpicos de Río de Janeiro en 2016 (Millington, Darnell \& Millington, 2018; Petrosillo et al., 2019), cuya sede brasileña supone el retorno del golf a las Olimpiadas desde 1904 (ver Figura 1). En cambio, en Estados Unidos parece que la cifra de golfistas está decayendo en los últimos años (Lyu \& Hwuang, 2017). Actualmente, el golf es considerado como el principal deporte en el mundo en términos de gasto económico (Han, Yoon \& Woods, 2016). 
Figura 1 - Campo Olímpico de Golf en Río 2016

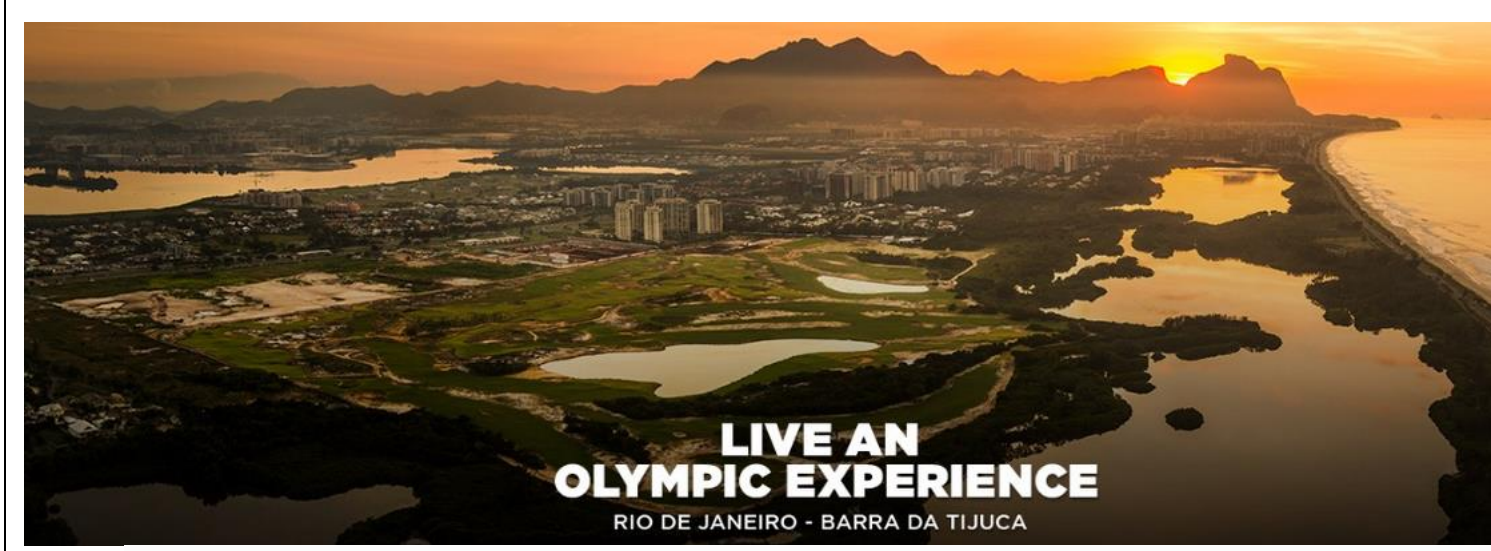

RIO DE JANEIRO - BARRA DA TIJUCA



Fuente: http://www.rioogc.com.br/en/home/

El Turismo de Golf puede ser una de las tipologías turísticas que genera más controversias sociales y políticas, sobre todo, debido al impacto medioambiental asociado a los campos de golf. El desarrollo del Turismo de Golf ha provocado estas controversias debido a que suelen basarse en proyectos de gran escala (Park et al., 2018). En este sentido, el golf tiene una huella medioambiental significativa, creciente y compleja (Minoli \& Smith, 2011). Los efectos nocivos relacionados con la actividad del golf abarcan la destrucción de los paisajes naturales, la sobre-explotación de recursos básicos como el agua, el abuso de fertilizantes, pesticidas y otros productos químicos, así como la contaminación, en general. Como subrayan Petrosillo et al. (2019), la mayoría de los estudios académicos realizados sobre los campos de 
golf han mostrado la confrontación intensa entre aquellos que están centrados en las consecuencias medioambientales adversas y aquellos otros que consideran que son un instrumento favorable para proteger la biodiversidad en un contexto urbano. No obstante, se pueden extraer también impactos socio-económicos positivos y negativos derivadas del Turismo de Golf.

La actividad del golf y la sostenibilidad son dos aspectos íntimamente relacionados. De hecho, como mencionan Han (2014), la cuestión ecológica es el talón de Aquiles del golf. Hoy en día, el proyecto, la construcción y el funcionamiento de un campo de golf deben estar supervisados por parámetros de sostenibilidad. Es más, la construcción de un campo de golf debe mejorar el valor medioambiental de los terrenos que ocupa (Espejo, 2004). Como indican Petrosillo et al. (2019), los campos de golf son considerados a menudo como 'infraestructuras verdes'. Todo ello requiere de una fuerte concienciación ecológica de los gestores de los campos de golf, pero también los jugadores de golf deben tener un comportamiento promedioambiental que esté en consonancia con este requerimiento.

El objetivo principal del presente estudio bibliométrico es identificar los trabajos realizados sobre Turismo de Golf durante las últimas décadas, adoptando una perspectiva de la sostenibilidad, ya que no cabe duda que es el mejor enfoque que se puede plantear considerando la estrecha relación que existe y debe existir entre ambos. Además, como sugieren Minoli y Smith (2011), los beneficios económicos y sociales del golf deben estar conciliados de manera que se permita continuar con su prosperidad y desarrollo. Por tanto, el futuro del Turismo de Golf está plenamente condicionado por su sostenibilidad medioambiental, económica y social. No obstante, el presente trabajo está más orientado hacia la materia de turismo y se trata de observar los estudios que analizan su conexión con los aspectos sostenibles.

\section{Revisión de la literatura}

Siguiendo a Liu et al. (2019), los estudios bibliométricos están basados principalmente en análisis cuantitativos de las publicaciones que pertenecen a un fenómeno específico. Se trata de un procedimiento eficiente para comprender cómo emerge y se desarrolla un campo de investigación (van Raan, 2005). Por tanto, es posible medir la evolución de un área de investigación específica a través de su producción científica, así como su productividad a lo largo de un período de tiempo concreto. Como sugieren Guzeller y Celiker (2019), el análisis bibliométrico puede examinar la estructura intelectual, las áreas de conocimiento, las áreas geográficas, los temas y métodos de investigación y los niveles de madurez de los tópicos de 
una disciplina o revista científicas. El análisis bibliométrico proporciona un enfoque más objetivo para explorar las tendencias y el rendimiento de la investigación, actuando como un método complementario a las revisiones de la literatura tradicionales (Jiang, Ritchie \& Benckendorff, 2017).

Varios autores han tratado de clasificar los distintos tipos de análisis bibliométricos. Así, por ejemplo, Tsang y Hsu (2011) sugieren que el análisis de publicaciones en revistas sobre turismo y hospitalidad pueden derivarse de tres amplias corrientes, como son: (1) los análisis de contribución de autoría e institucional, que son los más frecuentes y se refieren a la identificación de autores o instituciones, cuyo propósito principal es establecer algún tipo de ranking; (2) los análisis de los métodos de investigación, que se ocupan de examinar los métodos estadísticos aplicados dentro de una disciplina; y (3) los análisis de perfiles, que tratan de resumir los trabajos que han sido publicados, los tópicos cubiertos y los lugares de procedencia de las publicaciones.

Albacete, Fuentes y Haro-Domínguez (2013) adoptan una clasificación más precisa basada en cinco apartados, que se enumeran como sigue: (1) análisis institucional, que se realiza para medir el desempeño de universidades y centros de investigación que más contribuyen en el ámbito turístico; (2) análisis de autoría, que se lleva a cabo para la identificación de los académicos con más aportaciones en la investigación turística, así como los más citados; (3) análisis de publicaciones, que con ello se trata de identificar especialmente las publicaciones más prestigiosas en el ámbito turístico; (4) análisis de contenido, que se utiliza para evaluar el desarrollo de la investigación turística a través de las publicaciones; y (5) análisis de redes, que se basa en el estudio de la formación de redes entre investigadores en el ámbito turístico.

Por último, Koseoglu, Sehitoglu y Parnell (2015) realizan una clasificación más reciente y nos parece bastante completa sobre los estudios bibliométricos en turismo y hospitalidad, de manera que establecen seis categorías, que pasamos a enumerar con más detalle a continuación.

(1) Estudios sobre ranking y valoración de revistas científicas (e.g. Guzeller \& Celiker, 2019; Hall, 2011).

(2) Estudios sobre identificación de artículos, que cubren las contribuciones de autores, instituciones y regiones (e.g. Law, Leung, \& Buhalis, 2010; Zhao \& Ritchie, 2007).

(3) Análisis de contenido, que se dedican a observar las tendencias de investigación, el crecimiento de la producción científica, los temas tratados y las metodologías aplicadas 
(e.g., Koseoglu et al., 2016; Guo, Jiang \& Li, 2019; Khoo-Lattimore, Mura, \& Yung, 2019).

(4) Análisis de citas, que se ocupa de examinar la influencia de autores, artículos y revistas (e.g., García-Lillo et al., 2018; Jiang, Ritchie, \& Benckendorff, 2019).

(5) Análisis de la investigación realizada en países concretos (e.g. Koseoglu, Sehitoglu, \& Parnell, 2015; Kozak, Kozak \& Uysal, 2007; Tsang \& Hsu, 2011).

El presente estudio se ha centrado en la segunda categoría establecida por Koseoglu, Sehitoglu y Parnell (2015), ya que se ocupa de identificar los artículos de revistas científicas, así como a los autores e instituciones que han tratado la temática de estudio del Turismo de Golf desde una perspectiva de la sostenibilidad.

Hace relativamente poco tiempo que la investigación académica ha centrado su atención en el Turismo de Golf (Mason \& Moretti, 2015). Por ello, es lógico que no hayamos detectado ningún trabajo que realice una revisión exhaustiva sobre el tópico del Turismo de Golf, en general. Tan solo hemos encontrado dos estudios recientes que realizan una revisión bibliográfica detallada sobre algunos temas más o menos tangenciales en torno a este tópico, como son los trabajos de Pradas-García y García-Tascón (2017) y de Petrosillo et al. (2019). El primero de ellos analiza el papel que desempeña el greenkeeper dentro de los campos de golf, basándose en la revisión de 43 artículos seleccionados de la base de datos Scopus. El segundo trabajo realiza una revisión sobre el impacto medioambiental de los campos de golf a partir de 239 artículos encontrados en las bases de Scopus, ScienceDirect y Google Scholar.

\section{Metodología}

El proceso seguido en la búsqueda bibliométrica se ha basado teniendo en cuenta otros estudios previos realizados en el ámbito del turismo en general (v.gr. Myung, McClaren \& Li, 2012; López-Bonilla et al., 2017, 2018a, 2018b; Guo, Jiang \& Li, 2019; Niñerola, SánchezRebull \& Hernández-Lara, 2019), así como en el contexto específico del Turismo de Golf (Pradas-García \& García-Tascón; 2017; Petrosillo et al., 2019).

Primeramente, se ha elegido la base de datos Scopus y, en segundo lugar, se ha completado la búsqueda con la base de Web of Science. Ambas bases de datos son las de mayor amplitud, reconocimiento científico y difusión internacional. De este modo, se ha buscado, en primer lugar, el término "golf” en el título y, en segundo lugar, el término "golf touris*” en el título, abstract y keywords de los trabajos publicados en las revistas especializadas en turismo 
y hospitalidad. Se consideran todos los artículos encontrados en las revistas especializadas en turismo y hospitalidad y, a su vez, se revisan los contenidos de los demás artículos que se hallaron en el resto de revistas no especializadas para ver si encajan en la temática de estudio. Asimismo, se ha buscado la combinación de las palabras "golf" en el título y "touris*” en el título, abstract y keywords, realizando la selección de artículos que hayan sido filtrados con una serie de palabras en el abstract y keywords relacionadas con la temática de estudio, como son las siguientes: ecology, ecological, environment, environmental, impact, sustainable y sustainability.

Como indica Hall (2011), los análisis bibliométricos se centran principalmente en revistas académicas. Por ello, se han seleccionados exclusivamente aquellos artículos que se publicaron en "journals", descartando las demás opciones, como editoriales, libros y capítulos libros. Se ha decidido hacer el análisis de los últimos veintidós años porque hay muy pocos artículos anteriores y han tenido una escasa repercusión académica. La búsqueda de artículos se ha cerrado a finales de febrero de 2020. El análisis bibliométrico se ha realizado a partir de la información obtenida sobre las revistas en las que se han publicado estos artículos, los autores y sus afiliaciones institucionales, los años de publicación y el número de citas recibidas.

\section{Resultados y Discusión}

En el presente trabajo vamos a profundizar en la revisión de la literatura académica sobre los campos de golf vinculados a la actividad turística, ya que nos centraremos en el Turismo de Golf y en su estrecha relación con la sostenibilidad. La investigación sobre la sostenibilidad de los campos de golf ha sido amplia, pero es necesario distinguirla de la investigación sobre Turismo de Golf, que ha sido mucho más limitada. En el primer quinquenio del siglo XXI surge un gran interés por la investigación medioambiental de los campos de golf (Petrosillo et al., 2019), por lo que parece comprensible que el interés académico se haya trasladado al estudio del Turismo de Golf a partir del año 2006. No obstante, la investigación sobre golf se ha concentrado esencialmente en las cuestiones medioambientales, descuidando los aspectos económicos y sociales (Completo y Gustavo, 2014). En cambio, la investigación sobre Turismo de Golf se enfoca hacia una mayor diversidad de perspectivas sostenibles, incluso se podría decir que estos estudios están más inclinados a tratar la temática de la sostenibilidad desde enfoques económicos y sociales. Hemos revisado un período de tiempo muy amplio desde el año 1998 hasta la actualidad, recogiendo un total de 46 artículos 
publicados, lo cual sugiere que la investigación sobre Turismo de Golf y sostenibilidad ha crecido en la última década, pero se encuentra todavía en sus albores.

Los primeros estudios publicados sobre Turismo de Golf parecen estar orientados hacia las reacciones controvertidas que generan su desarrollo e implantación en distintos territorios en el mundo, como en Myanmar y Tailandia (Parnwell, 1998), en Hawai (Wyllie, 1998a, 1998b) o en Mallorca (Schmitt, 2000). Sin embargo, el estudio de Markwick (2000) se puede considerar como el primer artículo que define claramente las pautas de la investigación sobre Turismo de Golf desde una perspectiva de sostenibilidad. De hecho, este trabajo ha sido con diferencia el más citado hasta la fecha sobre esta temática (este tópico) y, por tanto, podemos considerarlo como el estudio seminal que impulsa esta nueva línea de investigación.

El interés académico por este tópico se ve impulsado a partir de 2006, en el que parece desempeñar un papel destacado el número especial de la revista Tourism and Hospitality Research, que publica varias ponencias presentadas en un congreso sobre golf organizado en el Algarve (Butler, 2006). La Figura 2 recoge los artículos publicados en cada año, observando una clara tendencia ascendente en la producción científica sobre Turismo de Golf y sostenibilidad.

Figura 2 - Número de artículos publicados en los últimos 20 años

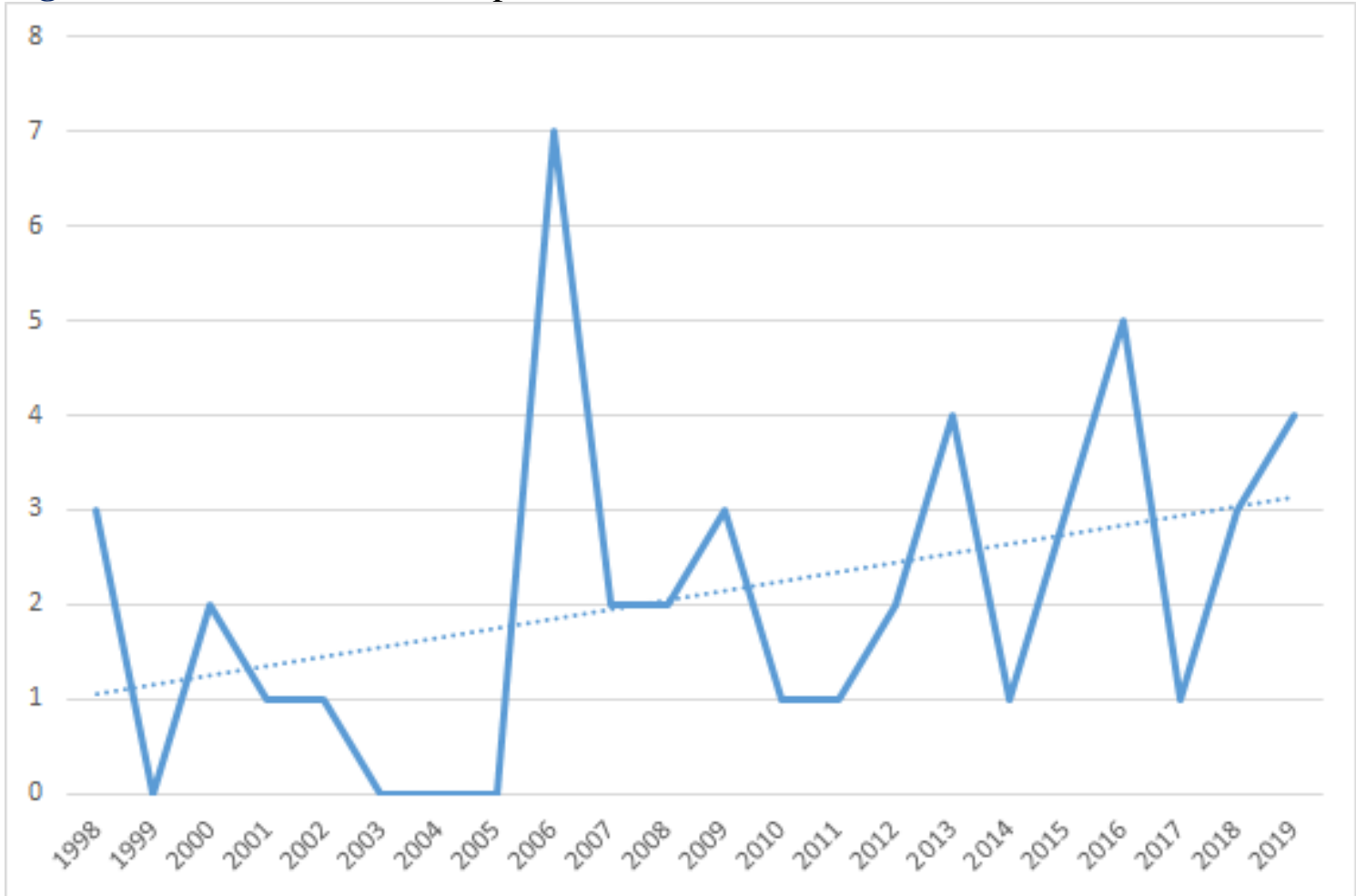

Fuente: Elaboración propia. 
En nuestro trabajo hemos encontrado 46 artículos publicados en revistas indexadas en Scopus y Web of Science, participando autores europeos en 32 artículos, que equivale al $70 \%$ del total, mientras los autores pertenecientes a países norteamericanos solo participaron en doce artículos, que supone el 26,1\%. En cambio, los trabajos publicados sobre los campos de golf proceden mayormente de Norteamérica, como indican Petrosillo et al. (2019). Por lo tanto, los estudios se enmarcan geográficamente en un contexto europeo, aunque hay escasez de trabajos publicados en países que son grandes receptores de turismo, como Francia, Italia y Alemania en Europa. En el mundo se echa en falta también trabajos publicados en otros países receptores de turismo como China, Tailandia y Japón. Los autores con una mayor producción científica pertenecen a instituciones españolas con la participación en 18 artículos $(39,1 \%)$, seguidos de autores estadounidenses, que participan en siete artículos (15,2\%), y de autores británicos, en seis artículos (13\%). La tabla 1 refleja estos datos.

Tabla 1 - Origen de autoría y lugar del estudio

\begin{tabular}{ccc}
\hline PAISES & $\begin{array}{c}\mathbf{N}^{\mathbf{0}} \text { artículos según } \\
\text { institución de autores }\end{array}$ & $\begin{array}{c}\mathbf{N}^{\mathbf{0}} \text { artículos según } \\
\text { contexto geográfico }\end{array}$ \\
\hline España & $18^{1}$ & 19 \\
USA & $7^{2,4}$ & 6 \\
Gran Bretaña & $6^{1,3}$ & 3 \\
Canadá & $3^{2}$ & 1 \\
Grecia & 3 & 3 \\
Australia & 2 & 1 \\
México & 2 & 2 \\
Portugal & 2 & 2 \\
Suecia & 2 & 1 \\
Alemania & 1 & 0 \\
Chipre & $1^{3}$ & 1 \\
Corea & $1^{4}$ & 2 \\
Croacia & 1 & 1 \\
Italia & 1 & 1 \\
Malta & 0 & 1 \\
Myanmar & 0 & 1 \\
Tailandia & 0 & \\
\hline Notas: ${ }^{1,2,3,4}$ nacionalidades de autores que comparten un artículo \\
Fuente: elaboración propia & &
\end{tabular}


En la tabla 2 se detalla los autores con mayor número de artículos publicados sobre la temática de estudio y que son responsables de 24 artículos, lo que significa algo más de la mitad del total de publicaciones analizadas (52,2\%). Todos ellos son autores europeos, salvo un autor canadiense y otro estadounidense. En concreto, hay 19 autores que tienen dos o más artículos publicados, con procedencia casi todos ellos de tres países: España, Portugal y Grecia. Se han publicado 18 artículos que se han enmarcado en contextos propios a estos tres países. Si se incluyen otros cinco estudios realizados en Malta (Markwick, 2000), Croacia (Jugovic, Grzinic \& Loncar, 2009) y España (Schmitt, 2000; Priestley, 2006; Górgolas, 2018), podemos decir que la mitad de los trabajos publicados se ubican en Europa del Sur. No obstante, entre todos los autores que han publicado sobre el tópico, es destacable mencionar también que uno de ellos, el profesor Butler, es uno de los principales investigadores en el ámbito de la sostenibilidad y el turismo en general, como se observa en el ranking de autores establecido por Niñerola Niñerola, Sánchez-Rebull y Hernández-Lara (2019).

Cabe mencionar que entre las publicaciones de autores españoles hay una elevada concentración de autores procedentes de universidades andaluzas. De hecho, casi todos los autores incluidos en la tabla 2 están adscritos a la Universidad de Huelva y a la Universidad de Sevilla, con tres y cuatro autores respectivamente cada una de ellas. Si sumamos otros dos trabajos firmados por autores del sur de España (Rodríguez et al., 2007; Górgolas, 2018), hay 14 de los 18 trabajos publicados por autores adscritos a instituciones españolas que pertenecen a la Comunidad Autónoma de Andalucía. Por su parte, se distingue en esta tabla 2 que hay cuatro autores de la Universidad del Algarve, pero han publicado conjuntamente dos artículos entre ellos.

Curiosamente, existe una extensión de territorio al suroeste de la Península Ibérica que dispone de una alta concentración de investigadores en el ámbito del Turismo de Golf, en cuya zona geográfica se unen las provincias españolas limítrofes de Huelva y Sevilla junto a la región portuguesa del Algarve. Las dos instituciones españolas están situadas en Andalucía occidental, por lo que se echa en falta una mayor presencia de investigadores procedentes de instituciones localizadas en provincias costeras donde ha habido un gran desarrollo de golf, como son las universidades de Almería, Cádiz y, sobre todo, Málaga.

Por lo tanto, se puede observar que los estudios sobre el Turismo de Golf se han concentrado, en gran medida, en zonas geográficas muy concretas, como son el Algarve, en Portugal, y, sobre todo, Andalucía, en España. En general, el gran interés académico por este tópico proviene de un espacio geográfico tan específico como es el sur de la Península Ibérica. 
Precisamente, la International Association of Golf Tourism Operators (IAGTO) ha situado ambas regiones entre los quince mejores destinos turísticos de golf en el mundo para 2020. En concreto, este ranking sitúa al Algarve en la primera posición mundial y a la Costa del Sol andaluza entre los siete destinos europeos que son mencionados.

Tabla 2 - Autores con mayor $\mathrm{n}^{\circ}$ de artículos

\begin{tabular}{cccc}
\hline AUTORES & INSTITUCIÓN & PAÍS & N $^{0}$ ARTS. \\
\hline Vargas-Sánchez, A. & Universidad de Huelva & España & 5 \\
López-Bonilla, J.M. & Universidad de Sevilla & España & 4 \\
López-Bonilla, L.M. & Universidad de Sevilla & España & 4 \\
Riquel-Ligero, F. & Universidad de Huelva & España & 4 \\
Briassoulis, H. & University of the Aegean & Grecia & 3 \\
Alves, I. & Universidad del Algarve & Portugal & 2 \\
Correia, A. & Universidad del Algarve & Portugal & 2 \\
Domínguez-Gómez, J.A. & Universidad de Huelva & España & 2 \\
Jönsson, E. & Lund University & Suecia & 2 \\
Lama, A.V. & Universidad de Sevilla & España & 2 \\
Martins, V. & Universidad Técnica de Lisboa & Portugal & 2 \\
Reyes-Rodríguez, M.C. & Universidad de Sevilla & España & 2 \\
Ramires, C. & Universidad del Algarve & Portugal & 2 \\
Salgot, M. & Universidad de Barcelona & España & 2 \\
Subtil, R. & Universidad del Algarve & Portugal & 2 \\
Tapias, J.C. & Universidad de Barcelona & España & 2 \\
Thilmany, D. & Colorado State University & USA & 2 \\
Videira, N. & Universidad Nueva de Lisboa & Portugal & 2 \\
Wyllie, R.W. & Simon Fraser University & Canadá & 2 \\
\hline
\end{tabular}

Fuente: Elaboración propia.

Por otro lado, existe una gran dispersión de las revistas en las que se han publicado los artículos, de manera que aparecen los 46 artículos en 35 revistas, siendo trece de ellas especializadas en turismo, otras tres revistas especializadas en deporte y una revista sobre deporte y turismo, que suman un total de 27 artículos entre ambas categorías. Hay otras nueve revistas especializadas en temas medioambientales, con un total de diez artículos. El resto de revistas están especializadas en geografía (4), economía (3) y en estudios regionales (2). Solo cinco revistas han publicado más de un artículo, que son: Tourism and Hospitality Research (5 artículos), Journal of Sustainable Tourism (4 artículos), Tourism Management (3 artículos), Journal of Sport and Tourism (2 artículos) y Sustainability (2 artículos). Hay más de la mitad 
de las revistas (19 en total) que están indexadas en Journal Citation Reports. En este sentido, las publicaciones en revistas sobre turismo han alcanzado por término medio un mayor factor de impacto que las revistas medioambientales. En la tabla 3 se recoge el listado de todas las revistas en las que los artículos han sido publicados.

Tabla 3 -Revistas donde se publicaron los artículos

\begin{tabular}{|c|c|c|}
\hline REVISTAS & $\begin{array}{l}\mathbf{N}^{\mathbf{o}} \\
\text { ART }\end{array}$ & $\begin{array}{c}\text { FACTOR } \\
\text { IMPACTO } \\
2018 \\
\end{array}$ \\
\hline Tourism and Hospitality Research & 5 & $\mathrm{SJR}=0,7(\mathrm{Q} 1)$ \\
\hline Journal of Sustainable Tourism & 4 & $\mathrm{IF}=3,4(\mathrm{Q} 1)$ \\
\hline Tourism Management & 3 & $\mathrm{IF}=6(\mathrm{Q} 1)$ \\
\hline Journal of Sport and Tourism & 2 & $\mathrm{SJR}=0,6(\mathrm{Q} 2)$ \\
\hline Sustainability & 2 & $\mathrm{IF}=2,6(\mathrm{Q} 2)$ \\
\hline Anatolia & 1 & $\mathrm{SJR}=0,4(\mathrm{Q} 2)$ \\
\hline Boletín de la Asociación de Geógrafos Españoles & 1 & $\mathrm{IF}=0,6(\mathrm{Q} 4)$ \\
\hline Cuadernos Geográficos & 1 & $\mathrm{SJR}=0,3(\mathrm{Q} 3)$ \\
\hline Current Issues in Tourism & 1 & $\mathrm{IF}=3,9(\mathrm{Q} 1)$ \\
\hline Economic research - Ekonomska istraživanja & 1 & $\mathrm{SJR}=0,4(\mathrm{Q} 3)$ \\
\hline Environmental Earth Sciences & 1 & $\mathrm{IF}=1,9(\mathrm{Q} 3)$ \\
\hline Environmental Engineering \& Management Journal (EEMJ) & 1 & $\mathrm{IF}=1,2(\mathrm{Q} 4)$ \\
\hline Environmental Management & 1 & $\mathrm{IF}=2,4(\mathrm{Q} 2)$ \\
\hline European Journal of Tourism Research & 1 & $\mathrm{SJR}=0,4(\mathrm{Q} 2)$ \\
\hline Geographische Zeitschrift & 1 & $\mathrm{SJR}=0,2(\mathrm{Q} 3)$ \\
\hline International Journal of Sport Management and Marketing & 1 & $\mathrm{SJR}=0,3(\mathrm{Q} 3)$ \\
\hline $\begin{array}{l}\text { International Journal of Sustainability in Economic, Social, and Cultural } \\
\text { Context }\end{array}$ & 1 & $\mathrm{SJR}=0,1(\mathrm{Q} 4)$ \\
\hline International Journal of Sustainable Development and Planning & 1 & $\mathrm{SJR}=0,2(\mathrm{Q} 3)$ \\
\hline International Journal of Urban and Regional Research & 1 & $\mathrm{IF}=2,8(\mathrm{Q} 1)$ \\
\hline Irrigation and Drainage & 1 & $\mathrm{IF}=1(\mathrm{Q} 4)$ \\
\hline Journal of Quality Assurance in Hospitality \& Tourism & 1 & $\mathrm{SJR}=0,5(\mathrm{Q} 2)$ \\
\hline Journal of Sport and Social Issues & 1 & $\mathrm{IF}=1,3(\mathrm{Q} 2)$ \\
\hline Journal of Sports Economics & 1 & $\mathrm{IF}=1,1(\mathrm{Q} 3)$ \\
\hline Journal of Travel and Tourism Marketing & 1 & $\mathrm{IF}=2,9(\mathrm{Q} 1)$ \\
\hline Journal of Travel Research & 1 & $\mathrm{IF}=5,3(\mathrm{Q} 1)$ \\
\hline Landscape Ecology & 1 & $\mathrm{IF}=4,3(\mathrm{Q} 1)$ \\
\hline Revista de Estudios Regionales & 1 & $\mathrm{SJR}=0,1(\mathrm{Q} 4)$ \\
\hline European Journal of Management and Business Economics & 1 & $\mathrm{SJR}=0,4(\mathrm{Q} 2)$ \\
\hline Revista Galega de Economía & 1 & $\mathrm{SJR}=0,2(\mathrm{Q} 4)$ \\
\hline
\end{tabular}




\begin{tabular}{lcc}
\hline Singapore Journal of Tropical Geography & 1 & $\mathrm{IF}=1,3(\mathrm{Q} 3)$ \\
Tourism Management Perspectives & 1 & $\mathrm{IF}=2,5(\mathrm{Q} 2)$ \\
Tourism Recreation Research & 1 & $\mathrm{SJR}=0,8(\mathrm{Q} 1)$ \\
Tourism Review & 1 & $\mathrm{IF}=1,1(\mathrm{Q} 4)$ \\
Tourist Studies & 1 & $\mathrm{IF}=1,4(\mathrm{Q} 3)$ \\
World Review of Science, Technology and Sustainable Development & 1 & $\mathrm{SJR}=0,2(\mathrm{Q} 3)$ \\
\hline
\end{tabular}

Fuente: Elaboración propia.

El número de citaciones de estos artículos es más bien escaso. El mayor número de citas recibidas lo ostenta el artículo publicado por Markwick (2000) en Tourism Management, con 99 citas hasta la fecha. Le sigue a continuación el artículo de Warnken, Thompson y Zakus (2001), publicado en Environmental Management (28 citas), y el artículo de Briassoulis (2007), publicado en Journal of Sustainable Tourism (22 citas).

En la tabla 4 se detallan todos los trabajos publicados y se distinguen los enfoques de sostenibilidad que se han adoptado en cada uno de ellos. Como sabemos, la sostenibilidad abarca distintas perspectivas que pueden resumirse en los aspectos medioambientales (med), económicos (eco) y sociales (soc). La tabla 4 trata de recoger de manera aproximada estas tres perspectivas o enfoques, observando que los temas que han sido más investigados se centran en el consumo de agua de los campos de golf (e.g. Del Campo, Molina \& Sales, 2006; Tapias \& Salgot, 2006; Rodríguez, Knox \& Weatherhead, 2007; Scott, Rutty \& Peister, 2018; Wurl, 2019), en la planificación y el desarrollo de proyectos sostenibles (e.g. Markwick, 2000; Priestley, 2006; Correia et al., 2006; Videira et al., 2006; Briassoulis, 2007), en la gestión medioambiental (e.g. Sullivan-Sealey \& Cushion, 2009 Vargas-Sánchez \& Riquel-Ligero, 2012, 2015; Riquel-Ligero \& Vargas-Sánchez, 2013; 2014) y en los impactos medioambientales positivos y negativos (e.g. Agrusa \& Tanner, 2002; Salgot \& Tapias, 2006; Domínguez-Gómez \& Vargas-Sánchez, 2016; Butler, 2019). 
Tabla 4 - Trabajos publicados, contexto y enfoque

\begin{tabular}{|c|c|c|c|}
\hline AUTORES & PAIS & CONTEXTO & ENFOQUE \\
\hline Parnwell (1998) & GB & Myanmar y Tailandia & soc \\
\hline Wyllie (1998a) & CAN & Hawaii (USA) & soc \\
\hline Wyllie (1998b) & CAN & Hawaii (USA) & soc \\
\hline Markwick (2000) & GB & Malta & med, eco, soc \\
\hline Schmitt (2000) & ALE & Mallorca (España) & soc \\
\hline Warnken et al. (2001) & AUS & Australia & med, eco \\
\hline Agrusa y Tanner (2002) & USA & Louisiana (USA) & eco \\
\hline Correia et al. (2006) & PORT & Algarve (Portugal) & med, eco, soc \\
\hline Del Campo et al. (2006) & ESP & Alicante (España) & med, eco \\
\hline Priestley (2006) & ESP & España & med, eco, soc \\
\hline Salgot y Tapias (2006) & ESP & España & med, eco, soc \\
\hline Tapias y Salgot (2006) & ESP & España & med \\
\hline Videira et al. (2006) & PORT & Algarve (Portugal) & med, eco, soc \\
\hline Wilson y Thilmany (2006) & USA & Colorado (USA) & eco \\
\hline Briassoulis (2007) & GRE & Europa Mediterránea & med, eco, soc \\
\hline Rodriguez et al. (2007) & $\mathrm{ESP} / \mathrm{GB}$ & España & med \\
\hline Lim y Patterson (2008) & AUS & Jeju (Corea) & eco \\
\hline Watson, Davies y Thilmany (2008) & USA & Colorado (USA) & eco \\
\hline Jugovic et al. (2009) & CROA & Istria (Croacia) & eco \\
\hline Sullivan-Sealey y Cushion (2009) & USA & Las Bahamas (USA) & med, eco \\
\hline Woodside (2009) & USA & USA & med, eco, soc \\
\hline Briassoulis (2010) & GRE & Creta (Grecia) & soc \\
\hline Briassoulis (2011) & GRE & Creta (Grecia) & soc \\
\hline López-Bonilla y López-Bonilla (2012) & ESP & Andalucía (España) & soc \\
\hline Vargas-Sánchez y Riquel-Ligero (2012) & ESP & Huelva (España) & med, eco \\
\hline Boukas y Ziakas (2013) & CHIP/GB & Chipre & med, soc \\
\hline Riquel-Ligero y Vargas-Sánchez (2013) & ESP & Huelva (España) & med, eco \\
\hline Villar-Lama y Fernández-Tabales (2013) & ESP & Andalucía (España) & med, eco \\
\hline Villar-Lama (2013) & ESP & Costa del Sol (España) & med, eco \\
\hline Riquel-Ligero y Vargas-Sánchez (2014) & ESP & Huelva (España) & med \\
\hline Flores et al. (2015) & MEX & México & med, eco \\
\hline Minoli, Goode y Smith (2015) & GB & Gran Bretaña & med, eco \\
\hline Vargas-Sánchez y Riquel-Ligero (2015) & ESP & Huelva (España) & med, eco \\
\hline Domínguez-Gómez y Vargas (2016) & ESP & Huelva (España) & eco, soc \\
\hline Jönsson (2016a) & SUE & Estocolmo (Suecia) & med \\
\hline Jönsson (2016b) & SUE & Aberdeenshire (Escocia) & soc \\
\hline
\end{tabular}




\begin{tabular}{lccc}
\hline Han, Yoon y Woods (2016) & COR/USA & Corea & soc \\
López-Bonilla y López-Bonilla (2016) & ESP & Andalucía (España) & soc \\
Domínguez-Gómez y González (2017) & ESP & Huelva (España) & soc \\
Gorgolas (2018) & ESP & Andalucía (España) & med, eco \\
López-Bonilla et al. (2018) & ESP & Andalucía (España) & soc \\
Scott et al. (2018) & CAN/USA & Ontario (Canadá) & med \\
Butler (2019) & GB & St. Andrews (Escocia) & med, eco, soc \\
Petrosillo et al. (2019) & ITA & Internacional & med, eco, soc \\
Wurl (2019) & MEX & Los Cabos (México) & med \\
López-Bonilla et al. (2020) & ESP & Andalucía (España) & soc \\
\hline
\end{tabular}

Fuente: elaboración propia.

\section{Consideraciones finales}

La investigación académica sobre Turismo de Golf ha experimentado un creciente interés en los últimos años. En el presente trabajo nos hemos centrado en el análisis bibliométrico de la investigación sobre Turismo de Golf desde el ámbito de la sostenibilidad. Creemos que es uno de los primeros estudios realizados sobre este tópico hasta la fecha. La importancia del Turismo de Golf es extraordinaria para el sector turístico por las grandes ventajas económicas que aporta. Sin embargo, es necesario tener muy en cuenta la influencia que puede ejercer también en la planificación y el desarrollo del territorio, tanto a nivel medioambiental como desde una perspectiva social. Por ello, este trabajo ha tratado de recoger las fuentes de investigación que más repercuten en la comunidad académica, a través de los artículos publicados en revistas científicas indexadas en la base de datos Scopus y Web of Science. Entre otros hallazgos, nos ha llamado la atención que exista una concentración muy elevada de estudios e investigadores localizados en zonas geográficas específicas en países mediterráneos y, más concretamente, en regiones que han adoptado el Turismo de Golf como una seña de identidad del propio destino turístico. Esperamos que el presente estudio sea de utilidad para descubrir el tipo de investigación que se ha realizado sobre este tópico, con idea de tener una referencia básica para poder continuar profundizando desde todas las perspectivas de la sostenibilidad.

La actividad del golf se desarrollo en un entorno físico y deben tener muy en cuenta el medio ambiente. Hoy en día es imprescindible adoptar medidas sostenibles en el desarrollo de los campos de golf. Existen muchos ejemplos de ello, como el Campo Olímpico de Golf de Río de Janeiro 2016, que se construyó bajo la premisa de un proyecto de recuperación ambiental basado en la vegetación propia del lugar. Los campos de golf deben ser considerados 
instalaciones verdes o ecológicas. Es evidente que el golf, el turismo relacionado con el golf y la sostenibilidad están estrechamente correlacionados. La investigación sobre los campos de golf se ha ocupado sobre todo de cuestiones medioambientales, aunque hay que precisar que la sostenibilidad no solo se ocupa de estos aspectos. En cambio, el Turismo de Golf ha prestado más atención a la triada de la sostenibilidad, siendo aspectos fundamentales tratados con frecuencia aquellos que tienen que ver con los impactos económicos y sociales, además de los medioambientales, como ocurre con el desarrollo local o regional, el aumento del empleo y la mejora de la calidad de vida de los habitantes o residentes del entorno geográfico.

El presente trabajo tiene diversas limitaciones que comentamos a continuación. En primer lugar, el estudio se ha basado en la búsqueda de artículos en las dos bases de datos internacionales más importantes en el mundo en la actualidad. Estas dos bases de datos tienen una gran cobertura de revistas indexadas y, además, son las que tienen un mayor reconocimiento académico. Sin embargo, este estudio no abarca otras fuentes de información. Asimismo, nos hemos centrado en la publicación de artículos, pero se ha descartado otros tipos de documentos publicados, como son, especialmente, los capítulos de libros y ponencias de congresos. En futuros estudios se podría realizar un análisis de contenido de los artículos a partir de las palabras clave, la metodología utilizada y la temática de estudio específica que se ha tratado. Asimismo, sería interesante plantear un análisis de redes para descubrir posibles conexiones entre las líneas de investigación existentes, los autores y los enfoques de sostenibilidad.

\section{Referencias}

Albacete, C.A., Fuentes, M.M., \& Haro-Domínguez, M.C. (2013). La investigación española en turismo con impacto internacional (1997-2011). Una perspectiva de la economía y la dirección de la empresa. Cuadernos de Economía y Dirección de la Empresa, v. 16, 17-28. doi.org/10.1016/j.cede.2012.05.003.

Agrusa, J., \& Tanner, J. (2002). The economic significance of the 2000 Buy.Com golf tournament on the Lafayette, Louisiana area. Journal of Sport \& Tourism, v. 7, n. 1, 6-24. doi.org/10.1080/10295390208718714.

Boukas, N., \& Ziakas, V. (2013). Exploring perceptions for Cyprus as a sustainable golf destination: Motivational and attitudinal orientations of golf tourists. International Journal of Sport Management and Marketing, v. 14, n. 1/4, 39-70. doi.org/10.1504/IJSMM.2013.060639.

Briassoulis, H. (2007). Golf-centered development in Coastal Mediterranean Europe: A soft sustainability test. Journal of Sustainable Tourism, v. 15, n. 5, 441-462. doi.org/10.2167/jost722.0. 
Briassoulis, H. (2010). "Sorry golfers, this is not your spot!": Exploring public opposition to golf development. Journal of Sport and Social Issues, v. 34, n. 3, 288-311. doi.org/10.1177/0193723510377314.

Briassoulis, H. (2011). Opposition to golf-related tourism development: an interpretivist analysis of an online petition. Journal of Sustainable Tourism, v. 19, n. 6, 673-693. doi.org/10.1080/09669582.2010.548559.

Butler, R.W. (2006). International Golf Congress: The present and the future of research. Tourism and Hospitality Research, v. 6, n. 3, 167-169. doi.org/10.1057/palgrave.thr.6050011.

Butler, R.W. (2019). Contributions of tourism to destination sustainability: golf tourism in St Andrews, Scotland. Tourism Review, v. 74, n. 2, 235-245. doi.org/10.1108/TR-12-2017$\underline{0197}$.

Completo, F., \& Gustavo, N. (2014). Golf tourism destination management: Looking for a sustainable demand: The case of Portugal. Journal of Management \& Sustainability, v. 4, n. 1, 142-153. doi.org/10.5539/jms.v4n1p142.

Correia, A., Barros, C.P., \& Silvestre, A.L. (2007). Tourism golf repeat choice behaviour in the Algarve: A mixed logit approach. Tourism Economics, v. 13, n. 1, 111-127. doi.org/10.5367/000000007779784489.

Correia, A., Videira, N., Alves, I., Ramires, C., Subtil, R., \& Martins, V. (2006). Tourism golf scenarios: The Algarve case. Tourism and Hospitality Research, v. 6, n. 3, 179-196. doi.org/10.1057/palgrave.thr.6050015.

Del Campo, F.J., Agulló, A.M., Aparicio, J., López, D.B., \& Izquierdo, R. (2018). Perception about the application of environmental management systems at golf course in Spain. Environmental Engineering and Management Journal, v. 17, n. 1, 53-62.

Del Campo, F.J., Molina, M.A., \& Sales, J.M. (2006). Sustainable limits for golf course development in a tourist destination. World Review of Science, Technology and Sustainable Development, v. 3, n. 3, 197-210. doi.org/10.1504/WRSTSD.2006.010221.

Domínguez-Gómez, J.A., \& González-Gómez, T. (2017). Analysing stakeholders' perceptions of golf-course-based tourism: A proposal for developing sustainable tourism projects. Tourism Management, v. 63, 135-143. doi.org/10.1016/j.tourman.2017.05.015.

Domínguez-Gómez, J.A., \& Vargas-Sánchez, A. (2016). Discussing the socio-economic impacts of tourism development projects based on golf courses: The perspective of local stakeholders. International Journal of Sustainable Development and Planning, v. 11, n. 3 , 365-374. doi.org/10.2495/SDP-V11-N3-365-374.

Dunlap, R.E., \& Van Liere, K.D. (1978). The “New Environmental Paradigm”. The Journal of Environmental Education, v. 9, n. 4, 10-19. doi.org/10.1080/00958964.1978.10801875. 
Espejo, C. (2004). Campos de golf y medio ambiente. Una interacción necesaria. Cuadernos de Turismo, v. 14, 67-112.

Flores, A., Vargas, E.E., \& López, L.J. (2015). Developing golf tourism in Mexico: Enrivonmental considerations. International Journal of Sustainability in Economic, Social, and Cultural Context, v. 11, n. 2, 35-48. doi.org/10.18848/2325-1115/CGP/v11i02/55154.

García-Lillo, F., Claver-Cortés, E., Úbeda-García, M., Marco-Lajara, B., \& Zaragoza-Sáez, P. C. (2018). Mapping the "intellectual structure" of research on human resources in the "tourism and hospitality management scientific domain. International Journal of Contemporary Hospitality Management, v. 30, n. 3, 1741-1768. doi.org/10.1108/IJCHM04-2017-0187.

Górgolas, P. (2019). The regulation of golf courses in the community autonomous of Andalusia: tourist interest or Business interest? Cuadernos Geográficos, v. 57, n. 1, p. 283304.

Guo, Y.; Jiang, J., \& Li, S. (2019). A sustainable tourism policy research review. Sustainability, v. 11, n. 3187, 1-16. doi.org/10.3390/su11113187.

Guzeller, C.O., \& Celiker, N. (2019). Bibliometrical analysis of asia pacific journal of tourism research. Asia Pacific Journal of Tourism Research, v. 24, n. 1, 108-120. doi.org/10.1080/10941665.2018.1541182.

Hall, C.M. (2011). Publish and perish? Bibliometric analysis, journal ranking and the assessment of research quality in tourism. Tourism Management, v. 32, n. 1, 16-27. doi.org/10.1016/j.tourman.2010.07.001.

Han, H. (2014). The norm activation model and theory-broadening: Individuals' decisionmaking on environmentally-responsible convention attendance. Journal of Environmental Psychology, v. 40, 462-471. doi.org/10.1016/j.jenvp.2014.10.006.

Han, H., Yoon, H., \& Woods, D.P. (2016). Role of environmental consciousness in golfers' decision formation in the traditional and screen-golf industries. Journal of Quality Assurance in Hospitality \& Tourism, v. 17, n. 3, 290-310. doi.org/10.1080/1528008X.2015.1077184.

Hudson, S., \& Hudson, L. (2010). Golf Tourism. Oxford: Goodfellow Publishing.

International Association of Golf Tourism Operators (2020). London:IAGTO. Disponible en: https://www.iagto.com/pressrelease/details/3bf83ba7-6443-4856-a79e-8f89116914a7. Recuperado en enero de 2020.

Jiang, Y., Ritchie, B.W., \& Benckendorff, P. (2019). Bibliometric visualisation: an application in tourism crisis and disaster management research. Current Issues in Tourism, v. 22, n. 16, 1925-1957. doi.org/10.1080/13683500.2017.1408574. 
Jönsson, E. (2016a). The nature of an upscale nature: Bro Hof Slott Golf Club and the political ecology of high-end golf. Tourist Studies, v. 16, n. 3, 315-336.

doi.org/10.1177/1468797615618306.

Jönsson, E. (2016b). Trump in Scotland: A study of power-topologies and golf topographies. International Journal of Urban and Regional Research, v. 40, n. 3, 559-577. doi.org/10.1111/1468-2427.12391.

Jugovic, A., Grzinic, J., \& Loncar, S. (2009). Macroeconomic legitimacy of investment in the development of golf tourism in Istria. Economic Research - Ekonomska Istraživanja, v. 22, n. 2, 66-85.

Khoo-Lattimore, C., Mura, P., \& Yung, R. (2019). The time has come: A systematic literature review of mixed methods research in tourism. Current Issues in Tourism, v. 22, n. 13, 1531-1550. doi.org/10.1080/13683500.2017.1406900.

Koseoglu, M.A., Rahimi, R., Okumus, F., \& Liu, J. (2016). Bibliometric studies in tourism. Annals of Tourism Research, v. 61, 180-191. doi.org/10.1016/j.annals.2016.10.006.

Koseoglu, M. A., Sehitoglu, Y., \& Parnell, J. A. (2015). A bibliometric analysis of scholarly work in leading tourism and hospitality journals: The case of Turkey. Anatolia, v. 26, n. 3, 359-371. doi.org/10.1080/13032917.2014.963631.

Kozak, N., Kozak, M., \& Uysal, M. (2007). Rankings of tourism and hospitality departments: A case of Turkey. Anatolia, v. 18, n. 2, 299-318. doi.org/10.1080/13032917.2007.9687207.

Latiesa, M., \& Paniza, J.L. (2006). Turistas deportivos. Una perspectiva de análisis. Revista Internacional de Sociología, v. 64, n 44, 133-149. doi.org/10.3989/ris.2006.i44.31.

Law, R., Leung, R., \& Buhalis, D. (2010). An analysis of academic leadership in hospitality and tourism journals. Journal of Hospitality \& Tourism Research, v. 34, n. 4, 455-477. doi.org/10.1177/1096348010370866.

Lim, C.C., \& Patterson, I. (2008). Sport Tourism on the Islands: The impact of an international mega golf event. Journal of Sport \& Tourism, v. 13, n. 2, 115-133. doi.org/10.1080/14775080802170346.

Liu, H., Liu, Y., Wang, Y., \& Pan, C. (2019). Hot topics and emerging trends in tourism forecasting research: A scientometric review. Tourism Economics, v. 25, n. 3, 448-468. doi.org/10.1177/1354816618810564.

López-Bonilla, J.M., Granados-Perea, C., \& López-Bonilla, L.M. (2017). Primera generación de autores con difusión internacional en la investigación turística española. Revista Española de Documentación Científica, v. 40, n. 3, 1-18. doi.org/10.3989/redc.2017.3.1399.

López-Bonilla, J.M., Granados-Perea, C., \& López-Bonilla, L.M. (2018). Producción científica española en turismo: un análisis de autoría basado en revistas internacionales con 
alto impacto y visibilidad. Cuadernos de Turismo, v. 41, 343-367.

doi.org/10.6018/turismo.41.327061.

López-Bonilla, J.M., Granados-Perea, C., \& López-Bonilla, L.M. (2018). Autores prolíficos líderes en la investigación turística española. Transinformaçao, v. 30, n. 1, 39-50.

doi.org/10.1590/2318-08892018000100004.

López-Bonilla, J.M., \& López-Bonilla, L.M. (2012). Environmental orientation in tourism: The RTEO scale. Current Issues in Tourism, v. 15, n. 6, 591-596. doi.org/10.1080/13683500.2012.657615.

López-Bonilla, L.M., \& López-Bonilla, J.M. (2016). From the new environmental paradigm to the brief ecological paradigm: A revised scale in golf tourism. Anatolia, v. 27, n. 2, 227236. doi.org/10.1080/13032917.2015.1100128.

López-Bonilla, J.M., Reyes-Rodríguez, M.C., \& López-Bonilla, L.M. (2018). The environmental attitudes and behaviours of European golf tourists. Sustainability, v. 10, n. 2214, 1-16. doi.org/10.3390/su10072214.

López-Bonilla, J.M., Reyes-Rodríguez, M.C., \& López-Bonilla, L.M. (2020). Interactions and relationships between personal factors in pro-environmental golf tourist behaviour: A gender analysis. Sustainability, v. 12, n. 1, 332, 1-19. doi.org/10.3390/su12010332.

Lyu, S.O., \& Hwang, J. (2017). Saving golf courses from business troubles. Journal of Travel \& Tourism Marketing, v. 34, n. 8, 1089-1100. doi.org/10.1080/10548408.2017.1285742.

Markwick, M.C. (2000). Golf tourism development, stakeholders, differing discourses and alternative agendas: The case of Malta. Tourism Management, v. 21, 515-524. doi.org/10.1016/S0261-5177(99)00107-7.

Mason, M.C., \& Moretti, A. (2015). Antecedents and moderators of golf tourists' behavioural intentions. An empirical study in a Mediterranean destination. EuroMed Journal of Business, v. 10, n. 3, 338-359. doi.org/10.1108/EMJB-10-2014-0033.

Millington, R., Darnell, S.C., \& Millington, B. (2018). Ecological modernization and the Olympics: The case of golf and Rio's "green" games. Sociology of Sport Journal, v. 35, n. 1, 8-16. doi.org/10.1123/ssj.2016-0131.

Minoli, D.M., Goode, M.M.H., \& Smith, M.T. (2015). Are eco labels profitably employed in sustainable tourism? A case study on Audubon Certified Golf Resorts. Tourism Management Perspectives, v. 16, p. 207-216. doi.org/10.1016/j.tmp.2015.07.011.

Myung, E., McClaren, A., \& Li, L. (2012). Environmentally related research in scholarly hospitality journals: Current status and future opportunities. International Journal of Hospitality Management, 31, 1264-1275. doi.org/10.1016/j.ijhm.2012.03.006.

Niñerola, A., Sánchez-Rebull, M.V., \& Hernández-Lara, A.B. (2019). Tourism research on sustainability: A bibliometric analysis. Sustainability, v. 11, 1377, 1-17. doi.org/10.3390/su11051377. 
Organización Mundial del Turismo (2019). Definiciones de turismo de la OMT. Madrid: UNWTO. https://doi.org/10.18111/9789284420858.

Park, J., Morrison, A.M., Wu, B., \& Kong, Y. (2018). Korean golf tourism in China: Place, perception and narratives. Sustainability, v. 10, 1055, 1-20. doi.org/10.3390/su10041055.

Parnwell, M.J.G. (1998). Tourism, globalisation and critical security in Myanmar and Thailand. Singapore Journal of Tropical Geography, v. 19, n. 2, 212-231. doi.org/10.1111/j.1467-9493.1998.tb00260.x.

Pastor, J.T., Del Campo, F.J., Vidal, F., Pastor, D., \& Agulló, A. (2015). Analysis of the efficiency of golf tourism via the Internet. Application to the Mediterranean countries. Current Issues in Tourism, v.18, n. 6, 595-608. doi.org/10.1080/13683500.2013.804806.

Petrosillo, I., Valente, D., Pasimeni, M.R., Aretano, R., Semeraro, T., \& Zurlini, G. (2019). Can a golf course support biodiversity and ecosystem services? The landscape context matter. Landscape Ecology, v. 34, 2213-2228. doi.org/10.1007/s10980-019-00885-w.

Pradas-García, M., \& García-Tascón, M. (2017). ¿Cuál es el papel del greenkeeper en un campo del golf? Podium: Sport, Leisure and Tourism Review, 6(3), 72-82. doi.org/10.5585/podium.v6i3.229.

Priestly, G.K. (2006). Planning implications of golf tourism. Tourism and Hospitality Research, v. 6, n. 3, 170-178. doi.org/10.1057/palgrave.thr.6050018

Ramírez-Hurtado, J.M., \& Berbel-Pineda, J.M. (2015).Identification of segments for overseas tourists playing golf in Spain: A latent class approach. Journal of Hospitality Marketing \& Management, v. 24, n. 6, 652-680. doi.org/10.1080/19368623.2014.934980.

RIOOGC (2020). Campo Olímpico de Golfe. Disponible en: http://www.rioogc.com.br/en/home. Recuperado en octubre de 2020.

Riquel-Ligero, F., \& Vargas-Sánchez, A. (2014). La legitimidad social de los campos de golf andaluces mediante prácticas ambientales y su relación con el desempeño: un estudio longitudinal. Revista Galega de Economía, v. 23, n. 2, 5-26. doi.org/10.15304/rge.23.2.2441.

Riquel-Ligero, F., \& Vargas-Sánchez, A. (2013). Environmental institutional pressures: An application to golf courses. European Journal of Management and Business Economics, v. 22, n. 1, 29-38. doi.org/10.1016/j.redee.2012.04.001.

Rodríguez, J.A., Knox, J.W., \& Weatherhead, E.K. (2007).Competing demands for irrigation water: Golf and agriculture in Spain. Irrigation and Drainage, v. 56, n. 5, 541-549. doi.org/10.1002/ird.317.

Salgot, M., \& Tapias, J.C. (2006).Golf courses: Environmental impacts. Tourism and Hospitality Research, v. 6, n. 3, 218-226. doi.org/10.1057/palgrave.thr.6050016. 
Schmitt, T. (2000). Quality tourism - a sustainable alternative to the development of tourism on Mallorca? Geographische Zeitschrift, v. 88, n. 1, 53-65.

Scott, D., Rutty, M., \& Peister, C. (2018). Climate variability and water use on golf courses: optimization opportunities for a warmer future. Journal of Sustainable Tourism, v. 26, n. 8, 1453-1467. doi.org/10.1080/09669582.2018.1459629.

Song, H., Chen, J.M., \& Chen, Y. (2020). Mediating and moderating effects in golf tourism:

Evidence from Hainan Island. Tourism Economics, 1-17. doi.org/10.1177/1354816620902331.

Sullivan-Sealey, K., \& Cushion, N. (2009).Efforts, resources and costs required for long term environmental management of a resort development: the case of Baker's Bay Golf and Ocean Club, The Bahamas, Journal of Sustainable Tourism, v. 17, n. 3, 375-395. doi.org/10.1080/09669580802275994.

Tapias, J.C., \& Salgot, M. (2006). Management of soil-water resources in golf courses. Tourism and Hospitality Research, v. 6, n. 3, 197-203. doi.org/10.1057/palgrave.thr.6050017.

Tsang, N.K.F., Hsu, C.H.C. (2011). Thirty years of research on tourism and hospitality management in China: A review and analysis of journal publications. International Journal of Hospitality Management, v. 30, 886-896. doi.org/10.1016/j.ijhm.2011.01.009.

van Raan, A. F. J. (2005). For your citations only? Hot topics in bibliometric analysis. Measurement: Interdisciplinary Research and Perspectives, v. 3, n. 1, 50-62. doi.org/10.1207/s15366359mea0301_7.

Vargas-Sánchez, A., \& Riquel-Ligero, F. (2015).Golf tourism, its institutional setting, and environmental management: A longitudinal analysis. European Journal of Tourism Research, v. 9, 41-56.

Vargas-Sánchez, A., \& Riquel-Ligero, F. (2012). Influence of the institutional context on the performance of golf courses, considering the natural environment. Environmental Engineering \& Management Journal, v. 11, n. 11, 2001-2012.

Videira, N., Correia, A., Alves, I., Ramires, C., Subtil, R., \& Martins, V. (2006). Environmental and economic tools to support sustainable golf tourism: The Algarve experience, Portugal. Tourism and Hospitality Research, v. 6, n. 3, 204-217. doi.org/10.1057/palgrave.thr.6050013.

Villar-Lama, A. (2013). Marketization of Mediterranean coastal landscape in Andalusia: The paradigmatic case of Costa del Sol and golf courses. Revista de Estudios Regionales, v. 96, 215-242.

Villar-Lama, A., \& Férnandez-Tabales, A. (2013). Diagnóstico y perspectiva territorial del golf en Andalucía: entre la cualificación turística y el desarrollismo inmobiliario. Boletín de la Asociación de Geógrafos Españoles, v. 62, 357-378. 
López-Bonilla, J.M., López-Bonilla, L.M., \& Reyes-Rodríguez, M.C. (2021, jan./abr.). Análisis bibliométrico sobre turismo de golf y sostenibilidad

Warnken, J., Thompson, D., \& Zakus, D. (2001). Golf course development in a major tourist destination: Implications for planning and management. Environmental Management, v. 27, 681-696. doi.org/10.1007/s002670010179.

Watson, P., Davies, S., \& Thilmany, D. (2008).Determining economic contributions in a recreational industry: An application to Colorado's golf industry. Journal of Sports Economics, v. 9, n. 6, 571-591. doi.org/10.1177/1527002508318595.

Wilson, J., \& Thilmany, D. (2007). Golfers in Colorado. Journal of Travel \& Tourism Marketing, v. 20, n. 3-4, 127-144. doi.org/10.1300/J073v20n03_09

Woodside, A.G. (2009). Applying systems thinking to sustainable golf tourism. Journal of Travel Research, v. 48, n. 2, 205-215. doi.org/10.1177/0047287509332335.

Wurl, J. (2019). Competition for water: Consumption of golf courses in the tourist corridor of Los Cabos, BCS, Mexico. Environmental Earth Sciences, v. 78, n. 24, 674. doi.org/10.1007/s12665-019-8689-y.

Wyllie, R.W. (1998a). Hana revisited: development and controversy in a Hawaiian tourism community. Tourism Management, v. 19, n. 2, 171-178. doi.org/10.1016/S02615177(97)00109-X.

Wyllie, R.W. (1998b). Not in our backyard. Opposition to tourism development in a Hawaiian community. Tourism Recreation Research, v. 23, n.1, 55-64. doi.org/10.1080/02508281.1998.11014820.

Zhao, W., \& Ritchie, J.R.B. (2007). An investigation of academic leadership in tourism research: 1985-2004. Tourism Management, v. 28, n. 2, 476-490. doi.org/10.1016/j.tourman.2006.03.007. 